\title{
Desarrollo de competencias genéricas en estudiantes que se desempeñan como tutores pares en la universidad
}

Development of Generic Competences in Students Who Work as Peer Tutors in the University

Desenvolvimento de competências genéricas em estudantes que se desempenham como tutores pares na universidade

\section{Raúl Bustos-González* id orcid.org/0000-0002-2363-1919}

\footnotetext{
Artículo de investigación

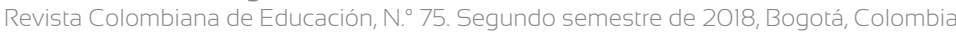

doi: 10.17227/rce.num75-8103

Para citar este artículo: Bustos-González, R. (2018). Desarrollo de competencias genéricas en estudiantes que se desempeñan como tutores pares en la universidad. Revista Colombiana de Educación, 75, 99-117.
}

\section{(c) $)(1) \Theta$}




\section{Resumen}

El presente artículo presenta una investigación que aborda el desarrollo de competencias genéricas en estudiantes que se desempeñan como tutores pares en la Universidad de Tarapacá. Se empleó una metodología mixta que considera la aplicación de una encuesta y la realización de tres grupos focales en los que participaron tutores pares, conformado cada uno dependiendo del número de semestres que han servido en su labor. Lo anterior permitió el descubrimiento de los significados asignados por los propios sujetos a los contenidos de la investigación. En conclusión, se relevó el trabajo tutorial como instancia no solo de apoyo a estudiantes de reciente ingreso, sino también como actividad que potencia la adquisición de competencias básicas y genéricas, como la empatía y el liderazgo, en estudiantes que desempeñen dicho rol.

\section{Palabras clave}

desarrollo de competencias; tutoría; aprendizaje mediante la práctica; enseñanza en equipo; apoyo pedagógico
Keywords

skills development; tutoring; learning through practice; team teaching; pedagogical support

\begin{abstract}
This article presents a research that deals with the development of generic competences in students who work as peer tutors at the University of Tarapacá. A mixed methodology was used, which considers the application of a survey and the realization of three focus groups in which peer tutors participated, each one formed depending on the number of semesters that they have served in their work. This allowed the discovery of the meanings assigned by the subjects themselves to the contents of the research. In conclusion, the tutorial work was revealed as an instance not only of support for recent students, but also as an activity that enhances the acquisition of basic and generic skills, such as empathy and leadership, in students who perform this role.
\end{abstract}

\begin{abstract}
Resumo
O presente artigo apresenta uma pesquisa que aborda o desenvolvimento de competências genéricas em estudantes que se desempenham como tutores pares na Universidade de Tarapacá. Utilizou-se uma metodologia mista que inclui a aplicação de uma enquete e a realização de três grupos focais nos que participaram tutores pares. Cada um dos grupos foi conformado segundo o número de semestres nos que os tutores têm realizado este trabalho. Isso permitiu a descoberta dos significados assignados pelos sujeitos aos conteúdos da pesquisa. Em conclusão, assinalou-se o trabalho tutorial como instância não só de apoio aos estudantes de novo ingresso, mas também como atividade que potencia a aquisição de competências básicas e genéricas, como a empatia e a liderança, em estudantes que desempenham este trabalho.
\end{abstract}

\section{Palavras-chave}

desenvolvimento de competências; tutoria; aprendizagem mediante a prática; ensino em equipe; apoio pedagógico 


\section{Introducción}

Hoy sabemos que la interrelación entre los estudiantes es un camino eficaz de construcción de aprendizajes, que permite alcanzar objetivos con rapidez y profundidad. Así, como señala el equipo de la Universidad Europea de Madrid, la tutoría entre pares "surge como una oportunidad de transmisión y adquisición horizontal del conocimiento, fomentando el trabajo autónomo de los estudiantes y ayudándoles a adquirir competencias que les serán útiles para afrontar diversos problemas a lo largo de sus vidas" (García, Gaya y Velasco, 2010, p. 120).

En el III Simposio Iberoamericano de Docencia Universitaria, realizado en 2004 en la Universidad de Deusto, se señalaron algunas de las ventajas de un sistema tutorial en la educación superior, particularmente en los aspectos formativos, informativos y de orientación (Guillamón et al., 2004)

Gabriela de la Cruz (2003) plantea que la tutoría se puede definir, por sus atributos característicos, como la relación entre un experto y un aprendiz, el que sea considerada como una estrategia de prevención y apoyo y que se traduzca en un esfuerzo cooperativo que involucra muchas otras acciones.

En diversos trabajos de la península ibérica se cuestiona al concepto primario de tutoría, orientado a resolver dudas de la asignatura, para dar lugar a una concepción más amplia de carácter orientador, que incluye además de la vida académica, expectativas sobre la vida profesional; es decir, con una concepción más integral. En España también los lineamientos oficiales han contribuido a orientar la función del acompañamiento:

Solo en algunas universidades privadas, que comienzan a surgir a raíz de la nueva Ley de Reforma Universitaria, empiezan a proponerse las tutorías como una mejora para sus alumnos. La Universidad San Pablo CEU, que fue la primera universidad privada homologada en España y que empieza su andadura en 1993, establece las tutorías como un derecho y un deber para los alumnos. (Fernández y Escribano, 2005, p. 16).

Esto es parte de lo que se percibe en la evolución de la mayoría de las propuestas de sistemas de tutorías, que justamente pasan de ser estrategias aisladas para convertirse en sistemas articulados (Guillamón et al., 2004). Fernández y Escribano (2005) afirman que el concepto de tutoría ha cambiado desde los años 1970, pasando de un sistema de servicios a un instrumento real para la orientación.

Otro ejemplo interesante es el proyecto tutor desarrollado por la Facultad de Filosofía y Ciencias de la Educación de la Universidad de Valencia, que divide las tutorías en dos grupos: académicas (vinculadas a la labor 
del docente), y personales de apoyo y seguimiento (que cumpliría una función informativa, de seguimiento académico e intervención formativa y de orientación).

En el contexto latinoamericano, hay algunos países que han registrado con mayor sistematicidad el desarrollo de iniciativas tutoriales y de acompañamiento a los estudiantes de educación superior. Entre estos países podemos mencionar a México, Venezuela, Colombia, Perú, Argentina y Chile (González, 2012).

Hernández y Vásquez (s.f.), en su documento Formación de tutores: una experiencia, dan cuenta del trabajo realizado por la Facultad de Ciencias Políticas y Sociales de la UNAM, proyecto instaurado desde 1972. Entre las conclusiones más destacadas están: la necesidad de un cambio de actitud hacia el trabajo colaborativo entre pares, la posibilidad de ser espacios virtuales de convivencia y la necesidad de que el tutor reflexione en torno a su práctica, generando discusión y reflexión sobre su labor, ejercicio del cual se recuperan ideas, y opiniones diversas en un ambiente de tolerancia y respeto, en el que la crítica permite enriquecer la práctica.

Por su parte, el programa de tutorías de la Universidad Quintana Roo (Moreno, s.f.) la caracteriza como una actividad flexible, oportuna y permanente, tendiente a motivar al estudiante a ser protagonista de su formación.

En virtud de lo anterior, han aparecido múltiples experiencias de mejora centradas en el campo de la función tutorial, como opciones institucionales en forma de programas de tutoría, que pueden diferenciarse entre tutorías ejecutadas por los académicos y las ejecutadas por tutores pares. Este último modelo es el de la Universidad de Tarapacá.

Podemos señalar como las principales diferencias entre la tutoría tradicional académica y la tutoría integral entre pares, de la que trata el presente trabajo, el carácter permanente de esta última y su orientación hacia la detección de necesidades y adquisición de habilidades (Gairín, 2012). La tarea del tutor, entonces, consiste en estimular las capacidades y procesos de pensamiento, de toma de decisiones y de resolución de problemas (Castaño, Blanco y Asensio, 2012).

Los programas que desean implementar un programa de tutorías académicas, como sucede en la mayoría de los casos, deben definir muy bien el tipo de competencias y conocimientos que han identificado como fundamentales para los estudiantes, incluyendo acá a las competencias genéricas, que serían

[...] necesarias para el adecuado desempeño profesional o académico independientemente del programa que hayan cursado [...]. Estas competencias se desarrollan entonces, 'mediante la interrelación entre la educación disciplinar, la educación general y las habilidades que los estudiantes han desarrollado y que traen a la educación superior'. 
(Klein, Benjamin, Shavelson y Bolus, 2008). Se trata de competencias transversales, que, aunque se desarrollen a partir de contextos específicos, pueden ser transferidas a otros. (Icfes, 2012, p. 7).

En las experiencias tutoriales desarrolladas en Argentina, la figura del tutor aparece como elemento central, por lo que la determinación de un perfil claro para quien deba cumplir estas funciones es clave. Laco y Guiggiani (2008) identifican ciertas características personales que debiera poseer un tutor, entre las que podemos señalar la perseverancia, la prudencia y la empatía.

En ese sentido, es necesario acentuar las habilidades permanentes que los estudiantes obtienen cuando trabajan como tutores de sus compañeros. Según Whitman (1988), el estudiante que actúa como tutor une la experiencia de un alumno (a lo largo de su carrera) con la experiencia de un docente en el sistema de instrucción (que él está asimilando).

Arbizu, Lobato y Del Castillo (2005) señalan como los principales beneficios de la tutoría entre pares, para los estudiantes/tutores: adquisición y desarrollo de competencias cognitivas y sociales, conducción y dinamización de pequeños grupos, formación en una función de asesoramiento y el que dicha función se transforma en un complemento de su currículum académico.

Según Castaño, Blanco y Asensio (2012) los tutores deberán tener desarrolladas prioritariamente la capacidad para comunicarse eficazmente, la escucha activa, el manejo de conflictos, trabajo en equipo y planificación del tiempo.

Un equipo de académicos de la Universidad Internacional de Florida preparó una encuesta que se envió a 126 antiguos tutores. Se analizaron las respuestas, y publicaron sus resultados (Hughes, Guillespie y Kail, 2010). Los extutores consideraron que la formación recibida y el trabajo realizado les dio beneficios tangibles que les permitieron mejorar su escritura, su confianza, su capacidad para escuchar y responder a los demás, y habilidades analíticas. Incluso, los profesores, que habían sido formados en la década de 1980 en la Universidad de Maine, informaron de que todavía utilizaban las destrezas y habilidades que desarrollaron cuando eran tutores.

Aparentemente, los tutores perciben que el desempeño de su labor los Ileva a superar temores al hablar en público y los estudiantes/tutores interesados en una carrera académica aprecian la exposición temprana a la enseñanza. Además, generan autorreflexiones sobre la aplicabilidad de la experiencia para sus carreras (Solomon y Crowe, 2001).

Otra experiencia similar es publicada por Peñaloza, Ordoñez y Restrepo (2014), en el contexto de la formación en medicina, evidenciando un impacto positivo en los estudiantes que integraron los equipos de tutores 
pares, especialmente en habilidades interpersonales y prácticas, lo que entregaría un método eficaz para proporcionar a los estudiantes las habilidades necesarias para ejercer como médicos en el futuro (Batchelder et al., 2010).

La Universidad tiene el compromiso de entregar los espacios y ejercer las acciones que permitan y promuevan el desarrollo de todos sus estudiantes, en el máximo de sus potenciales. Hoy existe la oportunidad de aprovechar los programas de tutores como una instancia, no solo de nivelación de competencias para estudiantes de reciente ingreso a la educación superior, sino además como un modelo de desarrollo de habilidades y competencias en alumnos de cursos avanzados.

Este hecho adquiere relevancia sustantiva, si se relaciona con el rol que una universidad pública debe cumplir desde una perspectiva regional, promoviendo la inclusión y el desarrollo de oportunidades a todos los estudiantes de los que es responsable. En este sentido, la atención de las estrategias institucionales debiera apuntar tanto a los estudiantes que presentan problemas para alcanzar el éxito académico, como a quienes aparecen con mayor ventaja y que muchas veces no pueden desarrollar a plenitud sus potencialidades en un contexto muchas veces poco motivante y carente de desafíos.

Los programas de tutoría entre pares, en potencia, pueden cumplir con la tarea aquí descrita.

Actualmente, la Universidad de Tarapacá cuenta con 82 tutores capacitados, lo que significa un grupo significativo de estudiantes en formación avanzada.

Así, proponemos que la labor tutorial promueva la adquisición y desarrollo de competencias genéricas en los estudiantes que se desempeñan como tutores pares en la dicha institución.

A fin de guiar la investigación, se responderá a las siguientes preguntas:

1. ¿Cuál es el perfil real del tutor/par de la Universidad de Tarapacá?

2. ¿Existe una diferencia significativa entre el perfil de los estudiantes de reciente ingreso en la Universidad de Tarapacá, y los que les prestan servicio de tutoría?

3. ¿Cuáles son las competencias que los tutores desarrollan y obtienen como resultado de su labor?

Para efectos de este artículo, y por la naturaleza de la labor del tutor, se usará la definición de competencias genéricas desarrollada por Sergio Tobón (2006), quien las define como aquellas que permiten un desempeño profesional idóneo y cuya formación promueve el aumento del grado de empleabilidad, la gestión y sostenibilidad laboral; favoreciendo de 
esta manera, la adaptación a los entornos laborales. Estas competencias no están ligadas a una profesión en particular y pueden adecuarse a los resultados y necesidades de cada institución o persona.

\section{Metodología}

Se entiende la propuesta metodológica como el sistema procedimental por el que una investigación produce información y la moviliza a distintos niveles de abstracción para generar conocimiento (Pelto y Pelto, 1978).

En virtud de lo anterior, se emplea una metodología mixta de investigación que se divide en dos etapas:

a) La elaboración del perfil particular del tutor par de la Universidad de Tarapacá, en base la aplicación de la encuesta CIDEU ${ }^{1}$ modificada, para determinar el Sistema de Apreciación del Desarrollo (SAD) que comprende los aspectos afectivos y cognoscitivos de cada estudiante, y que permite identificar factores inductores, ejecutores y de retroalimentación de la actividad, en este caso académica. La estructura del instrumento señala un índice promedio, medido de 0 a 1 , donde la mayor cercanía a 1 señala condiciones más favorables en el estudiante.

b) Para profundizar el análisis, se optó por una metodología cualitativa, en vista de enfrentar el problema desde el paradigma de la complejidad y poder dar cuenta a la multiplicidad de variables que interactúan en el fenómeno (Morin, 1995).

Si bien la perspectiva cualitativa no siempre ha sido considerada adecuada por parte de la comunidad científica (Aguirre, 1995), se adhiere a quienes afirman que este tipo de investigación ha generado las condiciones necesarias para lograr la comprensión contextualizada de los fenómenos educativos (Hymes, 1982).

Precisamente, gracias a la importancia dada al contexto real se producen los fenómenos educativos; además, se ha privilegiado una aproximación etnográfica a nuestro objeto de estudio. Este enfoque permitirá ver la realidad como una construcción que solo es factible de comprender a partir de los significados asignados por los sujetos que la han construido (Pérez, 1994). Al respecto, Jiménez (2007) señala que este tipo de investigación busca lo que ha llamado universales concretos alcanzados a través del análisis de casos particulares y de la comparación de ese caso con otros.

1 La encuesta CIDEU, que otorga el indicador SAD (Sistema de Apreciación del Desarrollo) fue desarrollada por Ernesto Figueredo y su equipo de trabajo. Esta se implementó sistemáticamente en varias instituciones de educación superior de Chile, de entre las que podemos mencionar la Universidad Autónoma de Chile, la Universidad de Humanismo Cristiano, Universidad de Tarapacá y Escuela de Aviación capitán Ávalos. 
Por otro lado, es conveniente reconocer el frágil equilibrio que exige la paradoja del estereotipo, que es al mismo tiempo "instrumento útil de previsión y control de la realidad, como también distorsión del conocimiento y obstáculo para la interacción, lo que nace del agravamiento de dos características intrínsecas del mismo: la generalización y la rigidez" (Navas, Holgado y Sánchez, 2009, p. 38).

Otra ventaja del enfoque propuesto se encuentra en el reconocimiento de la capacidad de acción de los sujetos (Feito, 1997). Así, lo normal en la investigación cualitativa es que el diseño del estudio evolucione a lo largo del proyecto. Este elemento distintivo de la investigación cualitativa obedece a la aspiración de representar la realidad y los diversos puntos de vista de sus participantes, los que están fuera de nuestro alcance al inicio de la investigación (Martín-Crespo y Salamanca, 2007).

En el mismo sentido, una de las condiciones que debe cumplir la investigación cualitativa es la fiabilidad, es decir, que un investigador que utilice los mismos métodos que otro debería llegar a idénticos resultados (Goetz y Lecompte, 1988, p. 214). Taft (1988) plantea que para generalizar de un caso individual a otros es necesario alcanzar una comprensión detallada de los eventos respecto a su contexto para poder extender interpretaciones a otros contextos. Esta contextualización es lo que permite hacer comparaciones (Ogbu, 1993).

La perspectiva desde la que se asume la presente investigación, la etnográfica, ha ganado espacio en la investigación educativa por su funcionalidad para comprender los fenómenos educativos y sus problemáticas, y a partir de ellos, proponer caminos de mejora (Jackson, 2001).

Si bien esta perspectiva es criticada por sus posibilidades de generalización, esto ocurre porque que se excluye su potencial heurístico en la contribución al conocimiento de un fenómeno, entendido este como la posibilidad de establecer nuevas relaciones y factores no atendidos anteriormente respecto al objeto de estudio, a través de la interpretación, que hagan necesario un replanteamiento del fenómeno estudiado (Leiva, 2009). Así, se podrían ofrecer nuevos datos, miradas y perspectivas que contribuyan a la mejora de la realidad educativa (Serra, 2004).

Por otro lado, Sturman (1988) propone una serie de estrategias para alcanzar credibilidad en este tipo de estudios, entre las que podemos señalar: la necesidad de que los procedimientos de recolección de datos deben ser explicados; los datos recogidos deben ser presentados y estar listos para reanálisis; las instancias negativas deben ser relatadas; los sesgos deben ser reconocidos, y deben diferenciarse las descripciones de las interpretaciones.

Parece indiscutible que adoptar una perspectiva etnográfica nos brinda la posibilidad de conocer, comprender y finalmente experimentar (Velasco y Díaz de Rada, 2009) de primera mano aquellos aspectos de interés 
investigativo que posibiliten responder nuestras preguntas de investigación. El interés del investigador se centra en construir una descripción contextualizada y no necesariamente en verificar, lo que no implicaría carecer de hipótesis o teoría inicial (Jiménez, 2013).

En resumen, los elementos claves de la investigación etnográfica (Hernández, Martínez y Montané, 2014) que sobresalen en la investigación emprendida son:

» La importancia otorgada a los relatos de las perspectivas y formas de comprensión de los participantes.

» La participación en una espiral de recopilación de evidencias, planteamiento de hipótesis y puesta a prueba de la teoría -que conduce a una mayor recopilación de evidencias-: se mantiene a lo largo del proceso de investigación.

»El enfoque en un caso particular, que se aborda en profundidad, para generar conocimiento.

En consecuencia, las investigaciones de este tipo incorporan una diversidad de técnicas para construir la información, de entre las que utilizaremos el grupo focal, con el objeto de lograr una doble aproximación: la del investigador y la de los participantes (Caballero, 2001; Jackson, 2001; Poveda, 2003).

Así, acudiremos a tres grupos focales:

a) Grupo 1: Tutores con un semestre de desempeño en su labor.

b) Grupo 2: Tutores con dos semestres de desempeño en su labor.

c) Grupo 3: Tutores con tres semestres de desempeño en su labor.

A partir de los grupos focales, es necesario desarrollar una aproximación a sus relatos que Moscoso (2014) Ilamó microcampos, que serían conexiones estructurales entre distintas perspectivas en torno a temáticas comunes, lo que se entiende teniendo presente las posiciones y las trayectorias sociales, así como las relaciones mutuas de los sujetos considerados en el estudio. Las temáticas son: perfil del tutor, diferencia con los tutorados y competencias desarrolladas en el desempeño de su labor.

Aquí es necesaria la reducción de los datos, la cual se hizo por separación en unidades, es decir, se realizó un análisis por fragmentación de la información en singularidades, lo que se conoce como categorización. Esta última se trabajó según criterios temáticos, porque permite más variedad de categorías y, por ende, de información valiosa.

Posteriormente, estos datos se codificaron para permitir una descripción y comprensión más profunda del objeto de estudio. Es preciso subrayar que se utilizaron codificaciones abiertas, con la emergencia de 
categorías ampliadas, redefinidas o modificadas, de acuerdo con lo que la misma investigación arrojaba. Las categorías de cada serie son mutuamente excluyentes, claras y replicables.

Luego, se empleó el análisis de contenido, porque se ajusta a los propósitos de la investigación, ya que permite estudiar, analizar y profundizar en las comunicaciones de manera sistemática. Este procedimiento es eficaz para las categorizaciones de datos verbales y para clasificar la información obtenida, lo cual facilita la creatividad y originalidad del investigador.

Para manejarlas con comodidad como expresiones-objeto, el análisis de expresiones orales se adelantó a partir de transcripciones escritas de estas. Por ello, en tanto se ocupa de expresiones escritas originalmente, como cuando se enfrenta a expresiones (originalmente) orales, se trabajó sobre textos.

Desde el punto de vista técnico, nos apoyaremos en el programa QDA Miner Lite, por su sencilla y eficiente aplicación para el análisis de contenido de carácter cualitativo.

Se trabajó con una muestra de 44 tutores institucionales, y para la elaboración del perfil de los estudiantes tutorados se encuestó al 86 \% de los estudiantes de reciente ingreso en la institución. A fin de contrastar el perfil de estudiantes con más de un año en la institución y de quienes se desempeñan como tutores, se encuestó a 507 estudiantes de segundo año de la Universidad de Tarapacá.

A fin de proporcionar confiabilidad al estudio se reconoce a una triangulación: informante/tiempo, diferenciando tutores con un semestre de labor, tutores con dos semestres de labor, tutores con tres semestres de labor. Estudiantes de primer año y estudiantes de segundo año.

Los instrumentos se aplican en fases sucesivas, y la obtención de datos se hace simultáneamente con su análisis y valoración.

\section{Determinación de perfil del tutor, de acuerdo con el diagnóstico institucional}

La primera fase de la investigación supone la elaboración del perfil de los estudiantes que se desempeñan como tutores pares, con el fin de tener un punto de partida en la distinción de las competencias desarrolladas por los tutores en su desempeño.

Se procedió, entonces, a la caracterización de los estudiantes de primer y segundo año, y de los tutores de la Universidad de Tarapacá, mediante la encuesta CIDEU, la cual se modificó para el cumplimiento de los fines del presente trabajo ${ }^{2}$.

2 La modificación consiste en homologar los instrumentos SAD1 (que se aplica a estudiantes de primer año) y SAD2 (que se aplica a estudiantes de segundo año), componentes del diseño original de la encuesta CIDEU, con el fin de permitir la comparación de los resultados entre ambos grupos. 
En cuanto a la encuesta a los de primer año, se logró cubrir a 1143 estudiantes de la matrícula 2014. En cuanto a los de segundo año, se encuestaron 507 estudiantes. Para el caso de los tutores, se trabajó una muestra de 44 tutores. En los tres casos, la muestra superó el $50 \%$ del universo a caracterizar.

En resumen, los tutores presentaron indicadores superiores a los de sus tutorados, especialmente en los aspectos de carácter emocional más que cognitivo, como las expectativas y motivaciones, aunque es interesante hacer notar que en ambos grupos este es el componente con menor desarrollo (figura 1).

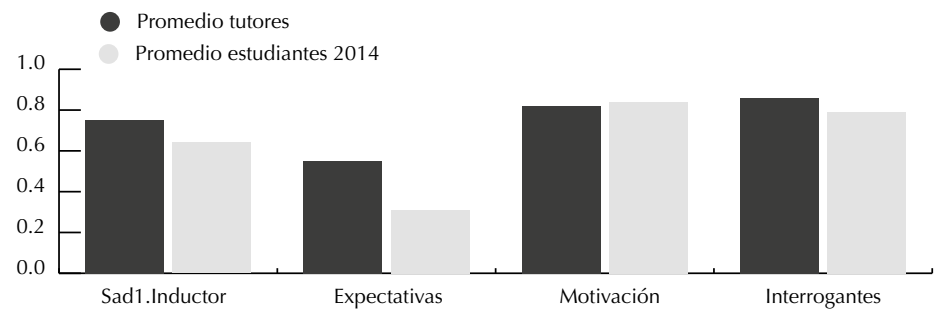

Figura 1. Comparación de factores inductores entre tutores y estudiantes ingreso 2014 (tutorados) $^{3}$

Fuente: elaboración propia.

Posteriormente se propone comparar el diagnóstico de los tutores y los estudiantes con más de un año en la institución, de acuerdo a los resultados obtenidos en esta cohorte para el indicador SAD2, que mide el desarrollo de competencias al segundo año de permanencia en la institución. En términos generales, el índice SAD es similar, aunque los estudiantes cohorte 2013 que se desempeñan como tutores el año 2014 presentaron un promedio SAD inferior al promedio de su cohorte al responder la encuesta como alumnos de primer año, en 2013 (figura 2).

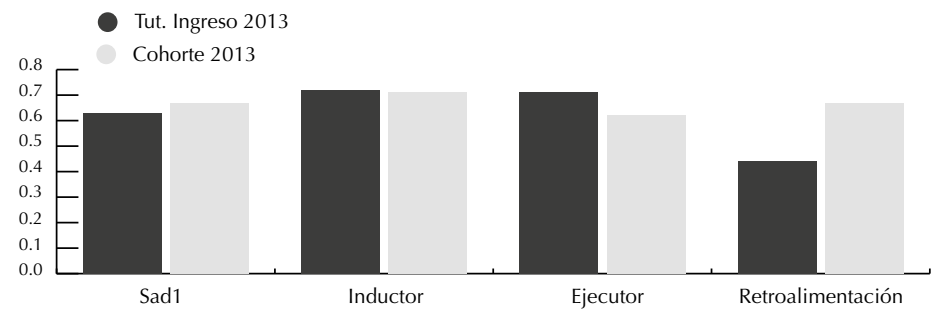

Figura 2. Comparación índice SAD entre cohorte 2013 y tutores miembros de dicha cohorte ${ }^{4}$

Fuente: elaboración propia. 


\section{Resultados: grupos focales con tutores ${ }^{5}$}

Los tutores en general en los grupos focales reconocen la existencia de un perfil que los diferencia de los tutorados (79 \% de los participantes señalan dicha situación). El grupo de tutores define su perfil como caracterizado principalmente por ser responsables, comprometidos y empáticos. Los tutores con un semestre en su labor la describen, en general, como sigue: "Si bien hay un perfil por carrera, pero creo que todos tenemos características en común, como empatía, solidaridad, sociabilidad, etc. Y estas características responden a un perfil" (Tutor, Masc., 20, 1).

Este hecho tiene ciertas consecuencias en lo cotidiano de los estudiantes que se desempeñan en esta labor: "El trabajo se nos sobrecarga, pero aprendo bastante" (Tutor, Fem., 19, 1).

En la misma línea, los tutores con tres semestres de desempeño (viejos) señalan con menor claridad la existencia de un perfil común entre los tutores, destacando solo dos elementos en este sentido: compromiso y afectividad. Este último elemento está ausente en los tutores con menor tiempo en su función.

Lo interesante es cotejar que el compromiso y la responsabilidad es un elemento del perfil presente en los grupos focales de manera transversal, sin importar el tiempo de desempeño de su labor. Sin embargo, se destaca que en tutores con dos semestres de trabajo aparece identificado el rol de mediador, desempeñado por los tutores, entre estudiantes y docentes:

Creo que también una competencia podría considerarse, la mediación porque en el rol de tutor tenemos que jugar mucho ahí, como tú dices, estar al medio. Y mediar, tratar de dejar bien al profesor con el alumno $y$ al alumno con el profesor y eso es todo un trabajo. A veces me he encontrado en situaciones en que no he estado de acuerdo con la postura de un profesor, pero tengo que tratar de buscar la forma de que el alumno lo tome de otra manera y no transmitirle mi postura personal y es difícil. (Tutor, Fem., 20, 2).

Una vez determinada la existencia de un perfil general de tutores, que se diferencia de los estudiantes tutorados, corresponde identificar las competencias que los estudiantes tutores reconocen han desarrollado en el desempeño de su labor.

En este caso, el desarrollo de las competencias de empatía, liderazgo y personalidad (entendido por los tutores como la capacidad de dirigirse a grupos y profesores con soltura) aparecen en general destacadas por los tres grupos de tutores, aunque dependiendo de las circunstancias que 
enfrentan de manera particular en el desempeño de su labor, les lleva a reconocer otras más: "Nosotros nadamos contra la corriente y eso, en mi caso, me ha permitido desarrollar más tolerancia a la frustración y un nivel más avanzado de resiliencia" (Tutor, Masc., 23, 3).

Sin embargo, nuevamente el número de códigos se ve disminuido en la medida que el tutor lleva más tiempo en su tarea. En tutores con más tiempo en su labor (un año como mínimo) el reconocimiento de competencias desarrolladas es menor, pero más decidido y convencido: "Es difícil, pero el programa me ayudó a ponerme en el lugar de ellos" (Tutor, Fem., 22, 3); "Me ayuda a saber cómo hablar ante distintas personas" (Tutor, Fem., 21, 3).

Por otro lado, los tutores con más tiempo en su labor reconocen el valor que el trabajo como tutor le entrega a su formación profesional: "A nosotros nos educan para ejercer en las empresas. Ahora aprendimos a trabajar en el área" (Tutor, Masc., 20, 3); "Ahora sé mejor cómo desarrollarme con los pacientes. Ahora los pacientes confían en mí" (Tutor, Fem., 24, 3).

Como podemos observar en la figura 3, los estudiantes que se desempeñan como tutores pares destacan fundamentalmente el desarrollo de la responsabilidad como principal competencia alcanzada en el desempeño de su labor. En segundo lugar, pero bastante lejana a la competencia señalada primero, aparecen el compromiso y empatía. La primera de estas la podemos señalar como componente de la misma competencia de responsabilidad.

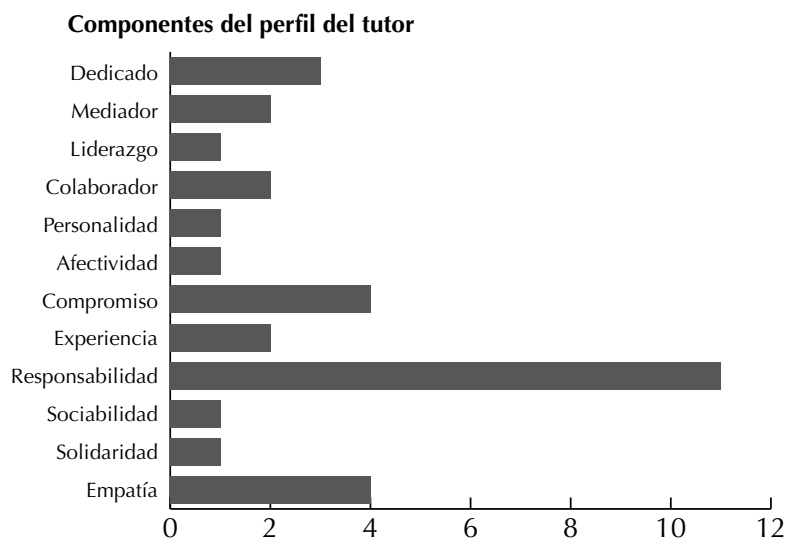

Figura 3. Frecuencia de códigos identificados por los tutores como características del perfil del tutor

Fuente: elaboración propia, a partir de tratamiento de trascripciones de grupos focales con QDA Miner. 
Por otro lado, la tabla 1 muestra la distribución de las características reconocidas por los tutores como parte de su perfil, en virtud del tiempo de desempeño de su rol. Llama la atención que los tutores con mayor tiempo en el desempeño de su tarea identifiquen menos características particulares.

Tabla 1. Frecuencia de elementos componentes del perfil del tutor, dividido por grupo de tutores

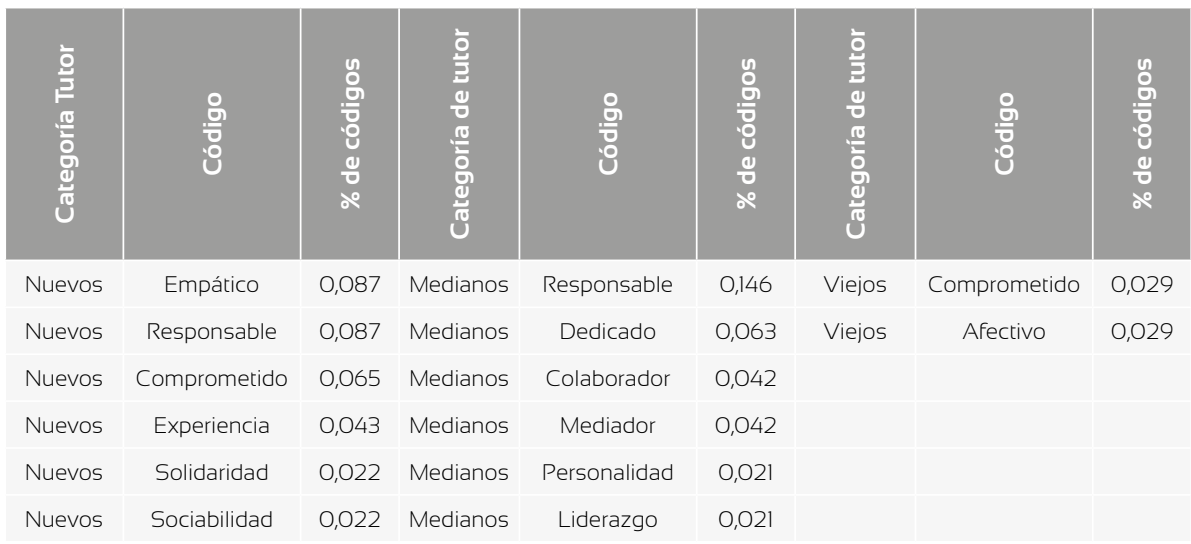

Fuente: elaboración propia, a partir de tratamiento de trascripciones de grupos focales con QDA Miner.

Es probable que el ejercicio cotidiano y permanente de su labor tiende a trivializar más el desarrollo de las competencias, destacando las que aparecen en el juicio del tutor como más recurrentes, a diferencia del tutor novato, que descubre un mundo nuevo que gatilla en él motivación:

Creo que he desarrollado más empatía, confidencialidad, que es super importante a la hora de acoger una problemática de una persona, y responsabilidad, lo cual se ha visto reflejado en mi desempeño como tutor y en mi desempeño como estudiante de la universidad.

Es probable que el mayor tiempo en el desempeño de su función pueda agudizar el juicio en el reconocimiento que dicha labor le ha otorgado a su formación.

Para complementar lo señalado anteriormente, se buscó que los tutores identificaran cuál de las competencias de su perfil son adquiridas como el resultado del desempeño de su labor. Así, se destaca el desarrollo de la empatía, liderazgo y personalidad (figura 4). 


\section{Competencias desarrolladas por los tutores en desempeño de su labor}

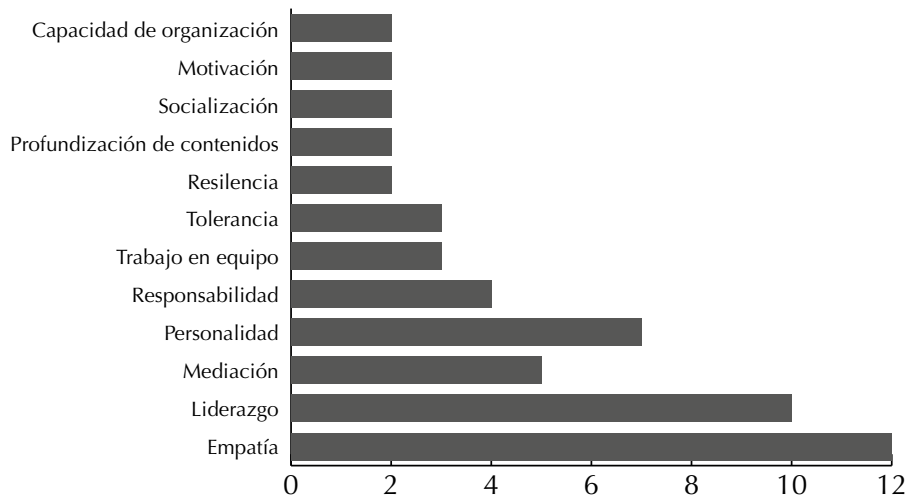

Figura 4. Frecuencia de códigos identificados por los tutores como competencias desarrolladas en el desempeño de su labor

Fuente: elaboración propia, a partir de tratamiento de trascripciones de grupos focales con QDA Miner.

\section{Conclusiones}

Los resultados de la presente investigación permiten señalar que los estudiantes que se desempeñan como tutores pares en la Universidad de Tarapacá reconocen en quienes desempeñan esta labor un perfil distintivo en relación a sus tutorados (estudiantes de reciente ingreso a la institución), y en algunos casos a sus compañeros de la misma generación de ingreso. Dicha diferencia es percibida por los mismos tutores como diferencia de madurez y habilidades comunicativas y organización.

Por otro lado, adquiere relevancia lo señalado por los tutores pares, en el sentido que las principales competencias que han desarrollado en el desempeño de su labor se encuentran en el ámbito de las competencias genéricas. Entre estas se destacan: empatía, liderazgo, personalidad y mediación.

Es de resaltar que el reconocimiento de las competencias desarrolladas en su labor se hace más preciso en los tutores que se han desempeñado por más tiempo en esa labor.

A partir de lo presentado en este trabajo, el trabajo tutorial se considera un elemento capaz de complementar la formación de los estudiantes destacados académicamente en sus carreras; además, favorece la integralidad de su crecimiento, potencia el dominio de habilidades útiles y necesarias en el desempeño de su futura actividad profesional, e incluso en otros aspectos de su vida, como la capacidad de ponerse en el lugar de los otros, coordinar y dirigir grupos, en la búsqueda siempre del bien común. 
Más adelante será necesario diseñar instrumentos que permitan medir ciertas competencias en particular para cotejar sus resultados, con las percepciones reconocidas por los tutores. No obstante, esa investigación posterior requiere de un punto de partida, que es lo que se pretende con este trabajo.

Por último, es necesario subrayar que el compromiso con una formación de calidad, inclusiva y con responsabilidad social es ineludible en una institución de educación superior pública y regional. En virtud de esto, cualquier iniciativa en dicho contexto que tienda a dar mayores oportunidades de desarrollo tanto a estudiantes vulnerables académicamente, como a quienes presentan ventajas relativas, adquiere relevancia en sí misma.

\section{Referencias}

Aguirre, A. (coord.). (1995). Etnografía. Metodología cualitativa en la investigación sociocultural. Barcelona: Marcombo.

Arbizu, F.; Lobato, C. y Del Castillo, L. (2005). Algunos modelos de abordaje de la tutoría universitaria. Revista de Psicodidáctica, 10(1), 7-22

Batchelder, A.J.; Rodrigues, C.M.; Lin, L.Y.; Hickey, P.M.; Johnson, C. y Elias, J.E. (2010). The role of students as teachers: four years' experience of a large-scale, peer-led programme. Med. Teach., 32(7), 547-551.

Caballero, Z. (2001). Aulas de colores y sueños: la cotidianeidad en las escuelas multiculturales. Barcelona: Ediciones Octaedro.

Castaño, E.; Blanco, A. y Asensio, E. (2012) Competencias para la tutoría: experiencia de formación con profesores universitarios. Revista de Docencia Universitaria, 10(2), 193-210.

De la Cruz, G. (2003). Evaluación de la tutoría: características y funciones del tutor desde las expectativas de los aspirantes a ingresar al posgrado. (Tesis inédita de Maestría). Universidad Pedagógica Nacional. México.

Feito, R. (1997). Odio la escuela. Una revisión de trabajos etnográficos en sociología de la educación. Revista Política y Sociedad, 24, 33-44.

Fernández, G. y Escribano, M. (2005). Las tutorías en la formación académica y humana de los alumnos de la Universidad San Pablo. Madrid: CEU UPCT. Recuperado de: http://metodos.upct.es/asepuma/comunicaciones/completas/605.pdf

Gairín, J. (2012). La actividad en el aula y la tutoría académica. Material del Máster en Formación y Gestión Universitaria. Departamento de Pedagogía Aplicada. Barcelona: Universitat Autònoma de Barcelona. 
García, M.; Gaya, M. y Velasco, P. (2010). Mentoría entre iguales: alumnos que comparten experiencias y aprendizaje. Actas XVI Jornadas de la Enseñanza Universitaria de la Informática Recuperado de: http://www.jenui2010.usc.es/actas_divididas/a15.pdf

Goetz, J. y Lecompte, M. (1988). Etnografía y diseño cualitativo en investigación educativa. Madrid: Morata.

González, B. (2012). Manual de formación de tutores. Documento de trabajo elaborado para la Asistencia Técnica en Certificación y Formación de Tutores y Profesores Guía. Arica: Universidad de Tarapacá.

Guillamón, C.; Quinquer, D.; Gairín, J.; Feixas, M. y Franch, J. (2004). Acciones tutoriales para la Universidad. III Simposio Iberoamericano de Docencia Universitaria. Bilbao, 21-24 de enero, Universidad de Deusto.

Hernández, J. y Váquez, J. (s.f.). Formación de tutores: una experiencia. Biblioteca Digital. Recuperado de: http://bibliotecadigital.conevyt. org.mx/colecciones/documentos/somece2002/Grupo4/Hernandez1.pdf

Hernández, F.; Martínez, S. y Montané A. (2014). Microetnografías y discontinuidad en una investigación sobre aprender a ser docente. En $\mathrm{H}$. Cárcamo (ed.), Making Of... Construcciones etnográficas de la educación (pp. 65-71). Recuperado de: http://e-spacio.uned.es/fez/eserv/ bibliuned:500383-IIICongresoEtnografia-1005/Documento.pdf.

Hughes, B.; Guillespie, P. y Kail H. (2010). What They Take with Them: Findings from the Peer Writing Tutor Alumni Research Project. The Writing Center Journal, 30(2),12-46.

Hymes, D. (1982). ¿Qué es la etnografía? En H. Velasco; F.G. García y A. Díaz de Rada (ed.), Lecturas de antropología para educadores (pp. 175-192) Madrid: Trotta.

Instituto Colombiano para la Evaluación de la Educación Superior (Icfes) (2012). Orientaciones para el examen de Estado de calidad de la educación superior Bogotá.

Jackson, P. (2001). La vida en las aulas. Madrid: Morata.

Jiménez S., L. (2007). Cuando estudiamos pertenencias étnicas en educación, ¿nos ayudan los modelos de análisis basados en categorías étnicas? EMIGRA Working Papers, 102. Recuperado de https://drive.google.com/file/d/OB8awT2JqXwtPYTk3ZTc3ZGQtZjliMy00OTc0LWlyMmMtZmJhMjgwNmMyYjcx/view?ddrp=1\&hl=en

Jiménez, F. (2013). Discursos y prácticas educativas en la escuela multicultural. (Tesis inédita de doctorado). Universidad Autónoma de Barcelona, Barcelona. 
Laco, L. y Guiggiani L. (2008). Programa Institucional de Tutorías. Un modelo integral. Buenos Aires, Argentina: Universidad Tecnológica Nacional.

Leiva, J.J. (2009). Educación y conflicto en escuelas interculturales: valores y concepciones pedagógicas del profesorado sobre convivencia y diversidad cultural. Revista Educación y Diversidad, 3, 107-149.

Martín-Crespo, M.C. y Salamanca, A. (2007). El muestreo en la investigación cualitativa. Nure Investigación, 27.

Moreno, T. (s.f.). Creación de una propuesta Institucional de tutorías para la enseñanza superior: El caso de la Universidad de Quintana Roo. Revista Electrónica de Educación 125. Recuperado de http://publicaciones.anuies.mx/revista/125/4/1/es/creacion-de-una-propuesta-institucional-de-tutorias-para-la-ensenanza

Morin, E. (1995). El pensamiento complejo. Madrid: Gedisa.

Moscoso, M. (2014). Acerca del enfoque (auto) biográfico aplicado a la investigación con niños y niñas. En H. Cárcamo (ed.) Making of... Construcciones etnográficas de la educación (pp. 93-98). Recuperado de http://e-spacio.uned.es/fez/eserv/bibliuned:500383-IIICongresoEtnografia-1005/Documento.pdf.

Navas, L.; Holgado, F. y Sánchez, A. (2009). Predicción de los estereotipos académicos ante los estudiantes inmigrantes. Horizontes Educacionales, 14(2), 37-47.

Ogbu, J. (1993). Etnografía escolar. Una aproximación a nivel múltiple. En A. Díaz de Rada, H. Velasco y F. García Castaño (eds.), Lecturas de antropología para educadores. El ámbito de la antropología de la educación y de la etnografía escolar (pp. 145-174). Madrid: Trotta.

Pelto, P.J. y Pelto, G.H. (1978). Anthropological Research: The Structure of Inquiry. 2a. ed. Nueva York: Cambridge University Press.

Peñaloza, I.; Ordoñez, K. y Restrepo A. (2014). ¿Impactó el programa de tutores pares a los tutores? (Tesis inédita para optar el título de especialista en Epidemiología). Colegio Mayor de Nuestra Señora del Rosario, Universidad CES. Bogotá.

Pérez, G (1994). Corrientes de investigación educativa. La etnometodología: aplicación a la educación cívico-social. En E. López- Barajs Zayas y J. Montoya Sáenz (ed.), La Investigación etnográfica, fundamentos y técnicas (pp. 41-68). Madrid: Universidad Nacional de Educación a Distancia. 
Poveda, D. (2003). Saberes sociolingüísticos en una clase multicultural. En D. Poveda (comp.), Entre la diferencia y el conflicto. Miradas etnográficas a la diversidad cultural en educación (pp. 67-98). Cuenca: Ediciones de la Universidad de Castilla-La Mancha.

Serra, C. (2004). Etnografía escolar, etnografía de la educación. Revista de Educación, 334, 165-176.

Solomon P. y Crowe J. (2001). Perceptions of student peer tutors in a problem based learning programme. Med. Teach., 23(2),181-186.

Sturman, A. (1988). Case study methods. En J.P. Keeves (ed.), Educational research, methodology, and measurement. An international handbook. (pp. 61-66). Oxford: Pergamon Press.

Taft, R. (1988). Ethnographic research methods. En J.P. Keeves (ed.). Educational research, methodology, and measurement. An international handbook (pp.71-75). Oxford: Pergamon Press.

Tobón, S. (2006). Las competencias en la educación superior. Políticas de calidad. Bogotá: Ecoe.

Velasco, H. y Díaz de Rada, A. (2009). La lógica de la investigación etnográfica. Un modelo de trabajo para etnógrafos de escuela. Madrid: Trotta.

Whitman, N. (1988). Peer teaching: to teach is to learn twice. ASHEERIC Higher Education Report. Washington, D.C.: ERIC Clearinghouse on Higher Education. 
